\title{
He I $5876 \AA$ Line in the Study of Chromospheric Activity in F-type MS Stars
}

\author{
R. J. García López ${ }^{1}$, R. Rebolo ${ }^{1}$, J. E. Beckman ${ }^{1}$, \\ and C. D. McKeith ${ }^{2}$ \\ ${ }^{1}$ Instituto de Astrofísica de Canarias, E-38200 La Laguna, Tenerife, Spain. \\ ${ }^{2}$ Department of Pure and Applied Physics, The Queen's University of \\ Belfast, Belfast BT7 1NN, Northern Ireland.
}

\begin{abstract}
We have measured the He I $D_{3}$ line, $\lambda 5876 \AA$, in 32 F-type young open cluster stars and in 4 field stars. Combining our results with previous observations of $\mathrm{D}_{3}$ we strengthen the following conclusions: (a) Sub-surface convective zones appear, on the main sequence, around F0 $(B-V \sim 0.3)$. (b) The stars between F0 and F5 $(0.3 \lessgtr B-V \lessgtr 0.42)$ are chromospherically "active" irrespective of age, which implies a heating mechanism not affected by evolutionary effects. In this range the activity in $\mathrm{D}_{3}$ and X-rays are uncorrelated. (c) For stars later than F5 the correlation among all the activity indices, chromospheric and coronal, shows a common energy source. Convective zones here should be deep enough to generate magnetic fields capable of supplying this energy, and also for braking the rotation.
\end{abstract}

\section{The $\lambda 5876 \AA D_{3}$ line of He I}

The utility of $\mathrm{He} \mathrm{I}_{3}$ as a chromospheric activity indicator was strongly suggested by studies such as that of Landman (1981) who showed the ratio of the line depth of $\mathrm{D}_{3}$ quiet.sun:plage to be $\sim 0.09$. This line is easier to observe in practice than the stronger He I IR line at $10830 \AA$, and although its excitation mechanism is disputed (Milkey et al., 1973; Zirin, 1975), and its equivalent width usually small ( $\$ 50 \mathrm{~m} \AA$ ), the advent of modern detectors and high resolution spectrographs has led to its use especially for the $\mathrm{F}$ spectral range, where indicators such as $\mathrm{Ca}$ II $\mathrm{H}$ and $\mathrm{K}$ emission have lost their sensitivity.

We observed the $\mathrm{D}_{3}$ line in F-type stars of two open clusters, 17 in the Hyades (age $\sim 6 \times 10^{8} \mathrm{yr}$ ) and 15 in the Ursa Major Group $\left(\sim 3 \times 10^{8} \mathrm{yr}\right)$ and in 4 field stars, all with previously known ages, metallicities and $\mathrm{Li}$ abundances. The observations were made with the QUBES spectrograph $\left(\lambda / \Delta \lambda \sim 6 \times 10^{4}, 43 \mathrm{~m} \AA /\right.$ pixel $)$ at the 
Cass. focus of the $4.2 \mathrm{~m}$ William Herschel telescope, La Palma. A typical S:N ratio was 200 .

\section{Flux in $D_{3} v$. spectral type}

We present in Fig. 1 the flux absorbed in $D_{3}: F\left(D_{3}\right)$ (computed from the equivalent width using neighbouring continuum flux from the appropriate Kurucz 1979 model) v. $(B-V)$ for all measured MS objects (classes V and IV-V) with $(B-V)<0.8$, both field and open cluster stars, from the present work and the literature (Lambert and O'Brien, 1983; Wolff and Heasley, 1984; Danks and Lambert, 1985; Wolff et al., 1985; Wolff et al., 1986; Wolff and Heasley, 1987). This plot confirms the following division of the $D_{3}-(B-V)$ parameter space (Wolff et al., 1986): (i) For $(B-V) \leqslant 0.3$ no measurable $\mathrm{D}_{3}$ is found, agreeing with previous results for this and other chromospheric and coronal indicators (Wolff et al., 1986; Schmitt et al., 1985; Walter et al., 1984). Models suggest (Vandenberg and Bridges, 1984) the "switch on" of convective envelopes around this $B-V$ value. (ii) All stars with $0.3 \lessgtr B-V \lesssim 0.42$ (known spectroscopic binaries removed) are " $\mathrm{D}_{3}$ active", with no apparent age dependence. In this enlarged sample selection effects appear unlikely. (iii) In the range $(B-V) \gtrless 0.42$ cluster stars (all young) show $\mathrm{D}_{3}$ activity, while some field stars do not. This is consistent with rotation-dependent (hence age-dependent) activity, stimulated by magnetic fields induced by rotation. Around the value $(B-V) \sim 0.4$ is where stellar envelope theory predicts a "switch on" of braking by stellar winds. $D_{3}$ appears as an excellent activity indicator for this effective temperature range.

The conclusions drawn here are strengthened when use is made of the Rossby number to parameterize magnetic activity (see García López et al., 1990).

\section{He I $D_{3}$ v. other chromospheric and coronal indicators}

(a) C IV $\lambda 1549 \AA$. There is a clear correlation between the IUE-measured emission flux in C IV $\lambda 1549 \AA$ (from the literature) and the absorption flux in $\mathrm{D}_{3}$, with no obvious dependence on spectral type or on whether the star is in a cluster. (b) $\mathrm{Ca}$ II $\mathrm{H}$ and $\mathrm{K}$. Similarly F'HK, measuring the $\mathrm{Ca} \mathrm{II} \mathrm{H}+\mathrm{K}$ emission core flux, correlates very well with $F\left(D_{3}\right)$ for those stars for which both are measurable. (c) X-ray luminosity. However a plot of the soft $\mathrm{X}$-ray luminosity in units of bolometric luminosity from Einstein satellite observations, against the equivalent widht of $\mathrm{D}_{3}$, divides into two zones: that with $(B-V)>0.44$, with a linear correlation coefficient $r$ of 0.89 , and that with $(B-V) \lessgtr 0.42$, where $r$ is only 0.33 . The difference in the behaviour of C IV $\lambda 1549 \AA$ and $\mathrm{Ca}$ II $\mathrm{H}$ and $\mathrm{K}$, on the one hand, and X-rays on the other is because the latter is a coronal activity index (see e. g. Dobson and Radick, 1989), while the other two, and $D_{3}$, are essentially chromospheric. 


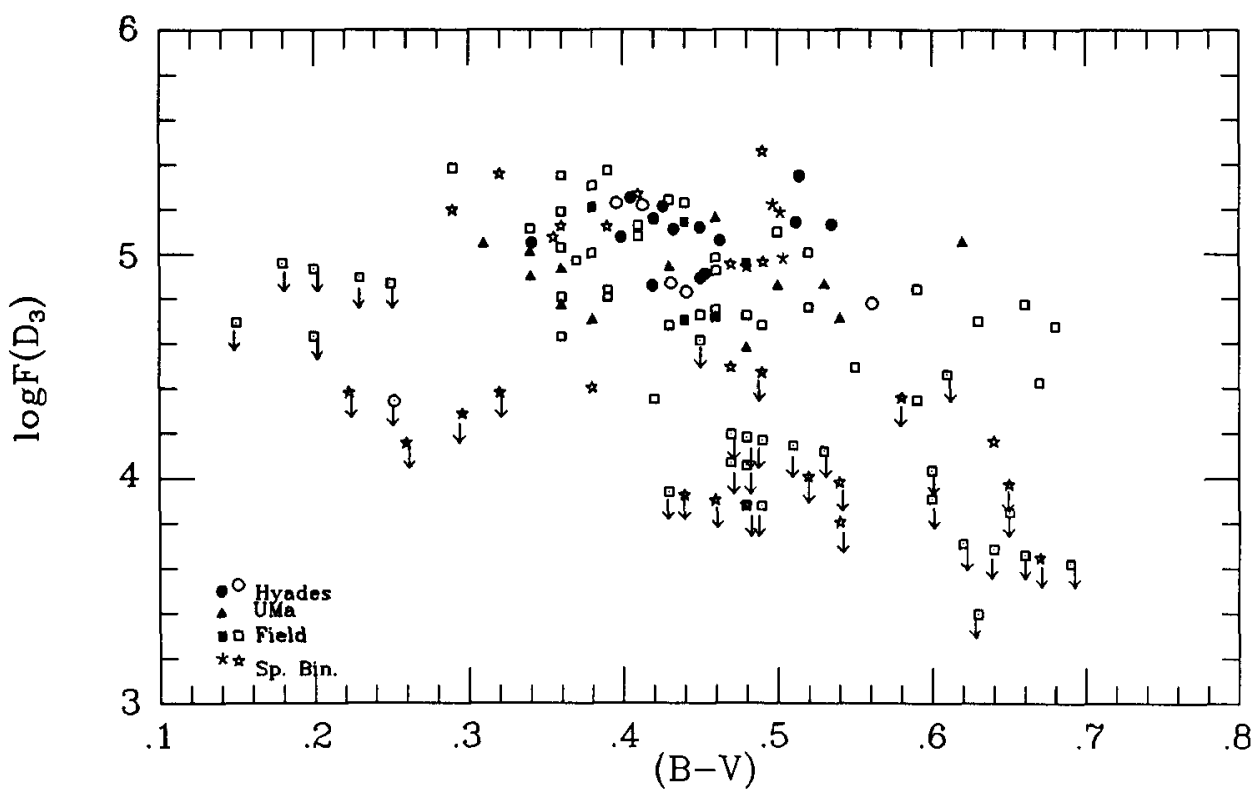

Fig. 1. Flux absorbed by the He $\mathrm{I}_{3}$ line (in $\left.\operatorname{erg} \mathrm{cm}^{-2} \mathrm{~s}^{-1}\right)$ v. $(B-V)$ for our stellar sample (filled symbols) and the literature (open symbols).

\section{References}

Danks, A. C., Lambert, D. L.: 1985, Astron. Astrophys. 148, 293

Dobson, A. K., Radick, R. R.: 1989, Astrophys. J. 344, 907

García López, R. J., Rebolo, R., Beckman, J. E., McKeith, C. D.: 1990, in Heidelberg Conference on Chromospheric and Coronal Heating, Springer-Verlag, in press

Kurucz, R. L.: 1979, Astrophys. J. Suppl. Ser. 40, 1

Lambert, D. L., O'Brien, G. T.: 1983, Astron. Astrophys. 128, 110

Landman, D. A.: 1981, Astrophys. J. 244, 345

Milkey, R. W., Heasley, J. N., Beebe, H. A.: 1973, Astrophys. J. 186, 1043

Schmitt, J. H. M. M. et al.: 1985, Astrophys. J. 290, 307

VandenBerg, D. A., Bridges, T. J.: 1984, Astrophys. J. 278, 679

Walter, F. M. et al.: 1984, Astrophys. J. 281, 815

Wolff, S. C., and Heasley, J. N.: 1984, Pub. A. S. P. 96, 231

Wolff, S. C., Heasley, J. N.: 1987, Pub. A. S. P. 99, 957

Wolff, S. C., Boesgaard, A. M., Simon, T.: 1986, Astrophys. J. 310, 360

Wolff, S. C., Heasley, J. N., Varsik, J.: 1985, Pub. A. S. P. 97, 707

Zirin, H.: 1975, Astrophys. J. Letters 189, L63 